\title{
A visual manipulation approach over constructive process management-interactive circles biosphere
}

\author{
Chi-Hsin Liang, Wei-Hao Haung, Chun-I Chen, and Teng-Wen Chang
}

\begin{abstract}
With the complexity of construction process, process information visualization and representation is often key research issues. Among those issues, when the problematic events occurred and need attentions of responsible persons, the notice methods are often applying multiple communication media such as vocal communication, SMS messages and pictures taken from the field in addition to emails, web pages and written documentation. Without fully integration among those media, the restriction as well as the advantage for each media shows.

With more advanced information technology appears now, the possibility of representation as well as the seamless integration becomes diverse and emergent in our daily life. Among those, a trend of human computer interfaces called ambient interfaces shows a promising direction for coordinating those media in a settle manor. Therefore, following three groups of researches-information technology in construction application, process information visualization and ambient interfaces, in this research we propose a prototype system called Interactive $\underline{\text { Circle }}$ Biosphere (ICBO) for testing the concepts for the problem we address. The implementation and components (interactive whiteboard and ambient lights) of ICBO are described in details. And, scenarios are described in terms of functionality and advantages of our prototype are included at the end of papers.
\end{abstract}

Index Terms-Ambient interface, construction information management, information visualization, intuitive manipulation.

\section{INTRODUCTION}

\section{A. Background}

TN the field of construction, many researches apply information technology onto the construction process itself. With the complexity of construction process, most of researches focus on the relationship and management of information flow. This is due to the lack of suitable representation for construction information. Nowadays, technology brings new possibilities in representing

C. H. Liang is with Graduate Institute of Architecture, National Chiao Tung University, Hsinchu, Taiwan. (e-mail: chi_hsin_liang@arch.nctu.edu.tw).

W. H. Haung is with Graduate Institute of Architecture, National Chiao Tung University, Hsinchu, Taiwan (e-mail: wayhow0411@msn.com).

C. I. Chen is with Graduate Institute of Architecture, Feng Chia University, Taichung, Taiwan (e-mail: chenchunyi1014@hotmail.com).

T. W. Chang is with Graduate School of Computational Design, National Yunlin University of Science and Technology, Douliou, Yunlin Taiwan (e-mail: tengwen@yuntech.edu.tw). information.

In addition to the information visualization, immediate feedback on the right time and right moment when situation occurred is another important factor. Currently, the information of some particular construction events is mainly reported back to the construction manager by devices that are designed for these usages. These devices are the mobile phone (including vocal communication, SMS messages, and picture taking), e-mail, web message, and in written documentation. Each device has its own advantages as well restriction. For example, even with silent mode, mobile phone still will invoke some communication interruption for the work of receivers. E-mail and written documentation can provide private channels for information, but it also ignores the concurrency needed for our problems. In addition, a simple but intuitive interface for awareness of the construction events might be a valid direction towards redefining the problems.

On the other hand, the development of Ubiquitous Computing technology brings a great opportunity of revolution in our daily life and environment. In the way of physical and virtual interaction, the daily human tactile senses the interaction with tangible interface proposes by a group of researches that would shift the information to the background and catches user's attention with ambient devices such as light, sound, air and water. This group of researches while will be discussed later in the paper inspires our research for interacting with visualized construction information.

\section{B. Problem and Objective}

In this paper, we will discuss the real time construction information visualization and how they interact with the users. We aim at the integration of the information that generated from the construction process with ambient interface design. Therefore, the objective of this research is to propose a prototype that would testify our concept of interaction and knowing its impacts on the construction information process.

\section{RELATED WORKS}

There are three related works that include a) Information technology application on construction, b) process information visualization, c) ambient interfaces. Each group of research 
inspires and challenges the solution of our research (as seen in Figure 1).

First group: Information Technology on Construction application has enormous development in applying IT onto construction problems. One thing in common in related to information visualization is that the researches often take advantages of network or precisely the Internet and databases. Every information occurred during construction processes will be transferred through network and provides a linked but diverse representation.

For example, Model (CARE-IS) [1] attempts to reorganize the industry and the information relations through Internet and database. In addition, [2] defines two types of interaction sub-processes (Information and Material process) in accordance with SADT modeling technique, then to address INFOMATE model about information process activities. While these researches provide significant strength on the information itself, inspiring ideas for representing constructive information is still a difficult problem. Representation such as 3D models is used to simulate the process of visual construction. Or adding the fourth dimension - time, it helps the designer to find the latent question in the design stage [3] . In addition, the state-of-the-art capture information technology such as laser scanners, [4] are used as information sensors to capture the real-time information on the spot.

Second group is process information visualization. This group is applying the information visualization theory and techniques on the problems of process. For example, [5] uses different level of visual relationship to present information flow on internet. By this manner, their research will reduce the visual load of users, and make people read easily with browsers. The problem is that even the virtual data is suitable for simulating people's sense organ but it cannot describe the information clearly and accurately.

In addition, [6] provides another view onto the similar problem, they analyze the salient features of information, and make proper correspondence with the vision elements of diverse zone. With interaction, the vision will be reproduced. This gives us an inspiration for solving our problems.

Third group is ambient interface. As mentioned from introduction, ambient interface provides a new way of

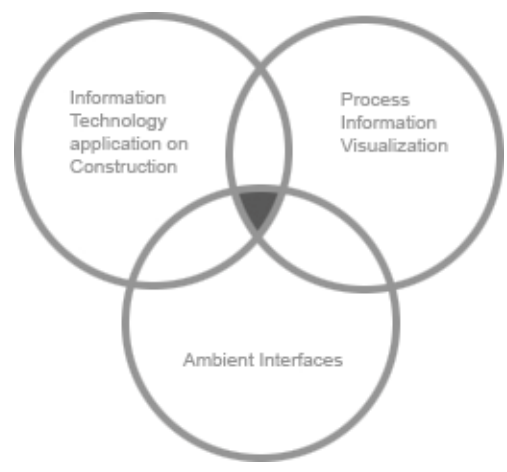

Fig. 1. Three groups of relevant works: Information technology application on construction, process information visualization, and ambient interfaces. un-interrupted awareness to the users. For example, [7] proposes one kind of ambient display orb to extend internet information to physical world. Such display can allow people to reduce the distraction from the information or work. And it allows users to easily read at a quick glance. By using ambient interfaces to visualize the information, the important information can be represented and interacted with ease and simplified manner.

There are four mainly elements in context that are important. They are time, location, identity and activity [8]. Among these, location is considered as the essential issue. For instance, [9-11] think that most of systems while based on the 2D or 3D coordination to represent the location, the real behaviors when people in context are missing. A kind of zoning system such as “Activity Zones" proposed by [12] is often used for defining the characteristics of location with certain classification.

With these three groups of research inspiration, we propose an implementation for testing our possible solution.

\section{IMPLEMENTATION}

What we propose in implementing the concept above is showing in several sections below.

\section{A. Approach}

Construction information awareness, is the bridge for user to aware the situation of the works instantly and directly. Therefore, we attempt to make an interface to improve the conventional tools in construction process, to let the information smarter and easier for user to acquire.

In order to achieve our goals, we decided to strengthen two parts of our interface, which contained the virtual and the physical parts. In virtual part of interface, it is important for user to aware the construction information generated by the system easily. While considering the awareness of information, visual effects are preferable rather than sound because of two reasons mentioned below.

1) The visual effects can be controlled more accurately than the sound.

2) It is more suitable for working space to make a public and privacy information at the same time by used the abstract patterns, showing in a synchronic interactive white board.

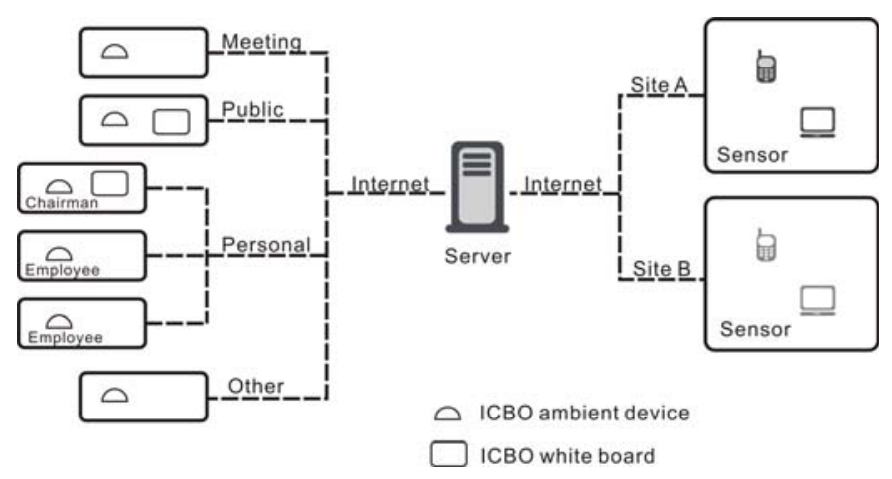

Fig. 2. The System infrastructure diagram: the integration of computing environment and physical context. 
And in physical part, the ambient and personalize environment is needed. The information will automatically find the context and person who needed it and use the ambient interface technology to present it. We used an ambient light device (as shown in Figure 3) to show the construction information because it can play a role of seamless communicator between virtual and physical world. Two main benefits for using such device as an information awareness device are:

1) Light is a direct way to let people aware the information instantly but not to interrupt people to a great extent than the other devices.

2) the feeling and representation of light can become personalize and context information by connecting each ambient device together.

\section{B. Computing Environment}

With the virtual and physical interfaces, our computing environment is comprised of three levels of relations for presenting the relationship of all construction elements as follow: a) Cases, b) the category of the construction (ex. Structure, apparatus), and c) the organization of construction (ex. grouting, hoop), and used the patterns to represent these information.

Two of the most important things in the process of the construction are 1) the achieved percentage of scheduled progress and 2) the quality of the building structures. So, we used the information as our key elements to show the condition of these patterns. The "red" color symbolized urgent situation in the entire world. Therefore, we used the brightness of red to show the different levels of urgent situation in construction. And in the whole world of culture, the " $X$ " is symbolized the massage of wrong things, in this reason we chose the glittered $\mathrm{X}$ to cover our ICBO when the construction didn't achieve the quality we expect.

At last, to think over the behaviors of people when getting the information from ICBO, they must take a clear understanding about the situation occurred and connect the responsible parties. Hence, the detail in the information section in this ICBO white board is shown with available media such as word phrases, photo and so on when the user choose the corresponded circle in this white board.

In addition, all the information mention above is carried synchronous through the Internet. The txt file format is used as the data storage format for updating the condition of the ICBO. The communication application (ex. msn, ftp etc...) can then be adapted to transfer a txt file and change the condition of our system.
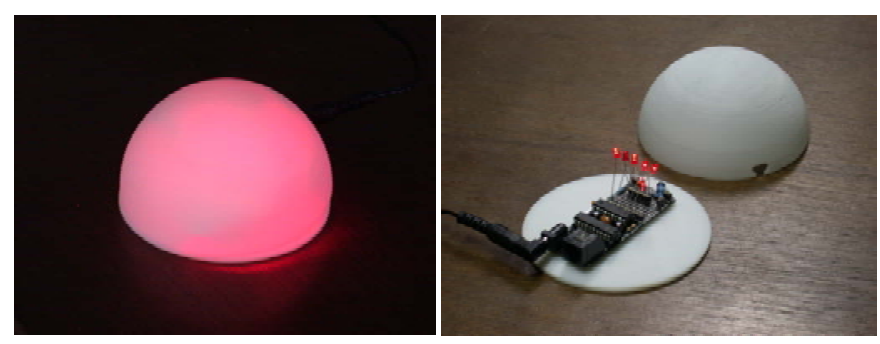

Fig. 3. The ICBO ambient light device and its hardware components

\section{Physical Context Integration}

Following the idea above, we need to seamless combine the device and the work environment. The ambient devices proposed should follow the activities of the space and strengthen the identity of context. For this reason, we divide the conventional workspace into four areas, 1) working space, 2) meeting space, 3) personal place and 4) others. We arrange the ambient devices into every personal place and other three areas mentioned above, and put the white board described before into the working space.

These physical device is activated just when people in these context. Like the patterns on the ICBO white board, we use the brightness of red light to show the different levels of urgent situation in construction. Depend on whom and where will need the information, the ambient light device will show the emergency information on the device in that user's personal area and context. When we aware the situation by the ambient devices, we can understand the detail information by the white board instantly.

\section{Implementation steps}

In virtual part, the classification of the information is depended on the cases invoked. One page shows the information in one case, and the page is divided into three areas: pattern area, text area and photo area. The metaphor of the pattern used to represent the construction information, which means:

1) The besieged circle: the relationship of the construction, ex. The structure section includes the units of grouting, hoop, and so on.

2) The radius: the proportion of budgets.

3) The position: current works are located in the center, and others are surrounding the page.

4) The animation: the radiate circle animation is representing the working situation.

5) The color: The color is representing different level of laggard construction.

6) The brightness $X$ : the construction didn't achieve the quality we expect.

In physical part, the user can touch the white board to switch

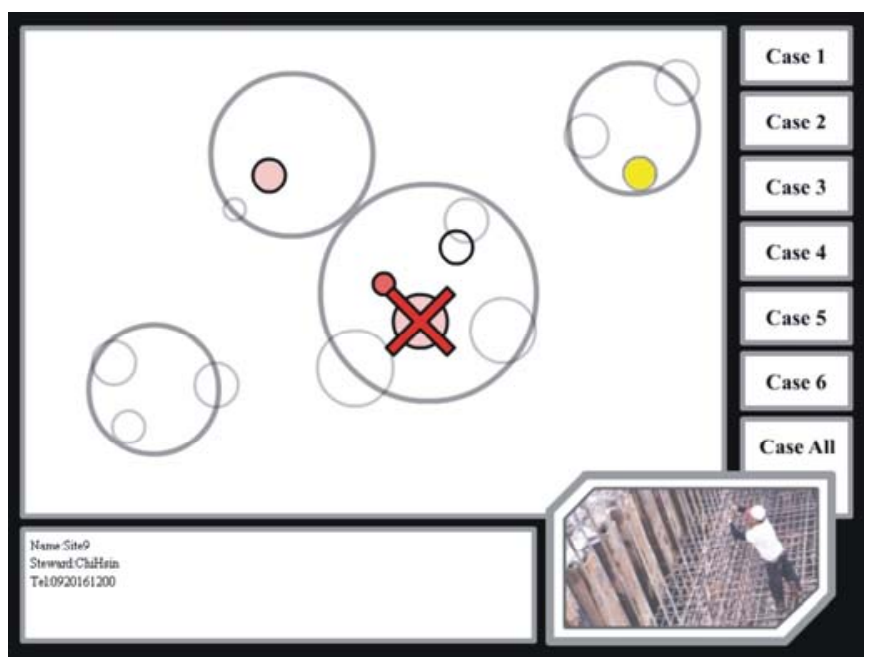

Fig. 4. The ICBO white board visualize interface. 


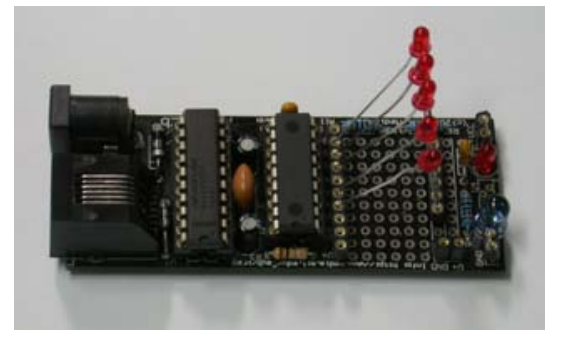

Fig. 5. The physical device: made by iRX with $16 \mathrm{~F} 84$.

the projects and construction fields. The touch sensor of white board is implemented by capacitance array and webcam. And the ambient light device described before is made by a half sphere, and used the iRX (as shown in Figure 5) board to control the Red LED, and displays the situation in physical world visually. The rule of the light is decided by the construction quality problem first, compare the most maximum problem and show in the ambient device by brightness lighting. If there is no construction quality problem, then the ambient device will compare all the case in white board, determined the most laggard construction and show the same level of red color on the white board.

The system infrastructure is based on the XML network protocol. We used the ICBO white board (as shown in Figure 4) as the server, and the ICBO ambient light devices as the clients, when the construction situation is changed, the server will determine the incoming information and make the decision to transfer the information to the corresponded clients. The system infrastructure is described in Figure 2.

\section{SCENARIO}

For showing how the ICBO works and possible effects it can provide, we describe four main different scenarios in different situations of interactions. Scenario one and two are for whiteboards. Scenario three and four are for ambient light.

\section{A. Scenario one}

Chi is an integrated information worker in construction site. He receives the diverse information from many construction projects everyday. There are many kinds of diagrams and text files on his desk (as shown in Figure 6). He felt the information very scattered and non systematic. He used most of time to coordinate the information and got bored on the work very quickly.

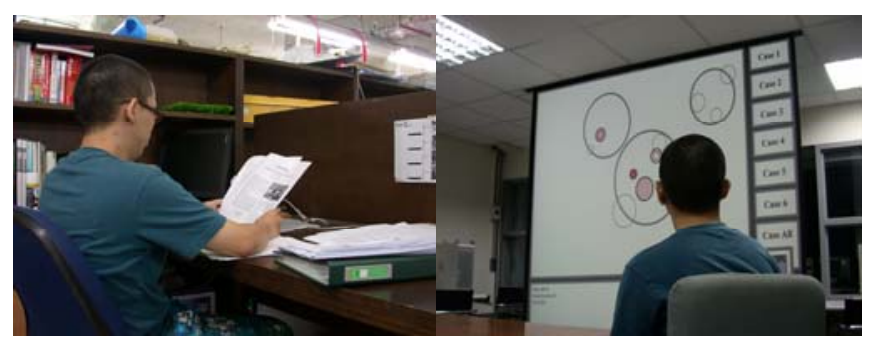

Fig. 6. The traditional interface of construction information reported and ICBO interface.
Through the ICBO white board, Chi doesn't need to face a lot of boring diagrams or documentation. The intuition visual patterns are used instead of these boring information, and through the project list, he can easily change the project he wants to understand, and clearly knows the relationship and category of the construction elements.

\section{B. Scenario two}

Wei is a construction management. His work is receiving the information from the construction site. He can't resolve multiple tasks at the same time while he is working on another project. When he is working to coordinate the diagram and data, the incoming phone calls often interrupt his thinking and the task he currently works (as shown in Figure 7). The result of effects not only let him restart his job but also waste the time in this project. Or, he will not handle the incoming phone calls and lost the timing of solving the problems.

Through the ICBO white board, Wei will not need to face various kinds of data. The ICBO integrates different kinds of data into the similar interface such that he does not need to deal with continuously phone calls. With the identical of information through Internet, Wei can even easily control his job by using this system remotely.

\section{Scenario three}

Chun, who is a manager, takes responsibility and extremely cares about the progress and the quality of construction. After inquiring the construction condition, he can be relieved and handles personal transaction. He felt the unceasing inquiry as a troublesome matter, thereupon stipulated the subordinate repayment information at fixed time. But such repayment can disturb his ordinary work. He extremely hoped, while handles personal transaction, to obtain the construction by some mode of the information, actually the matter that pondered to him does not create the disturbance.

By the physical interface of ICBO, Chun needs not constantly worry about the construction condition at the site, but he can still receive the right information passively at any time he wants (as shown in Figure 8).

\section{Scenario four}

Teng is the king of the construction industry. He spends almost all his time to social events with other celebrities and businessmen. The confidential information of each project cannot be shown in public even with emergency situations. Therefore, he must finish the current events, then to deal with the information of his company. It makes a lot of inconvenience

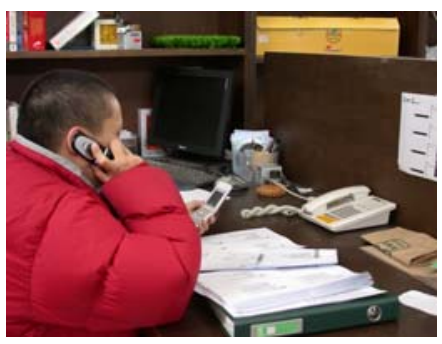

Fig. 7. The incoming phone calls often interrupt manager's thinking and current task. 


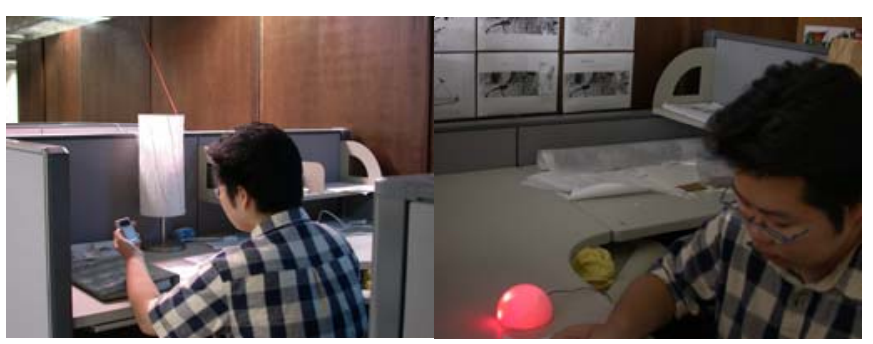

Fig. 8. The ICBO ambient device make information passively and continuous.

when he must communicate with his clients or anyone in the social events. He can't deal with the work and social event at the same time.

Through the ICBO ambient device, Teng can perceive the condition and know what if he needs to deal with when he is communicating with others (as shown in Figure 9). He does not need to finish the social event by using this ambient device.

\section{CONCLUSION}

In this research, we are more familiar with the abstract progress of information visualization; especially related to the basic visual elements (colors and shapes). We found that moving patterns (attraction and confliction) could arouse a person's perception more intense. Besides, there are many visualization factors with cognitive meanings (such as colors, transparencies and flashes) can apply on the same object.

\section{A. Significance}

By the means of interacting design, we propose a possible interface that could be used in the information stage in a construction process. The significance we have is to provide a seamless interface for dealing with diverse information with ubiquitous conditions. In addition, the exploration for better visualization patterns as well integrating them with network is also another advantage of our approach.

\section{B. Limitation and Future Study}

The ICBO system, under the circumstance that various sensors have collected construction information, computes and presents the visual information from working site. Therefore, for developing the ICBO system further, it is unavoidable to lead the scope of the research into the field that uses automatic sensors to collect construction information dynamically. On the other hand, for limited scope, the progress and quality of construction are the only two factors we have considered in this research. Different factors or information should be explored and integrated into ICBO for better understanding the problems we address.

\section{REFERENCES}

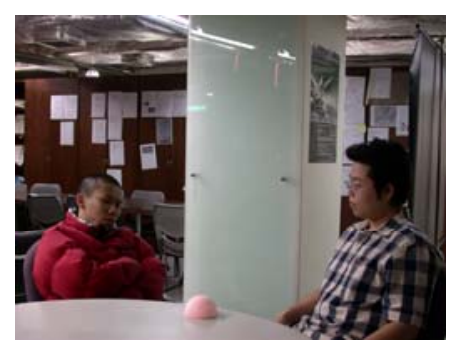

Fig. 9. The ICBO ambient device presents condition in the social events.
S. Mak, "A model of information management for construction using information technology," Automation in Construction, vol. 10, pp. 257-263, 2001.

B.-C. Björk, "Information Technology in construction: domain definition and research issues," International Journal of IT in Architecture, Engineering and Construction (IT-AEC), vol. 1, pp. 3-16, 1999.

K. McKinney, M. Fischer, and J. Kunz, "Visualization of construction planning information," in Proceedings of the 3rd international conference on Intelligent user interfaces. San Francisco, California, United States: ACM Press, 1998, pp. 135-138.

B. Akinci, J. Garrett, and M. Patton, "A Vision for Active Project Control using Advanced Sensors and Integrated Project Models," presented at Specialty Conference on Fully Integrated and Automated Project Processes, Virginia Tech, 2002.

J. Donath, H.-Y. Lee, D. Boyd, and J. Goler, "Loom2-Intuitively Visualizing Usenet," presented at CSCW 2001 (workshop), 2001. D. Boyd, H.-Y. Lee, D. Ramage, and J. Donath, "Developing Legible Visualizations for Online Social Spaces," presented at 35th Annual Hawaii International Conference on System Sciences (HICSS'02), Big Island, Hawaii, 2002.

P. Barry, U. Dekel, N. Moraveji, and J. Weisz, "Increasing contribution in online communities using alternative displays of community activity levels," presented at CSCW 2004, 2004. G. D. Abowd, A. K. Dey, P. J. Brown, N. Davies, M. Smith, and P. Steggles, "Towards a Better Understanding of Context and Context-Awareness," presented at 1st International Symposium on Handheld and Ubiquitous Computing (HUC’99), Berlin Heidelberg, 1999.

B. Johanson, A. Fox, and T. Winograd, "The Interactive Workspaces Project: Experiences with Ubiquitous Computing Rooms," IEEE Pervasive Computing, vol. 1, pp. 67-74, 2002. M. Beigl, "Special Issue on Location Modeling in Ubiquitous Computing," Personal and Ubiquitous Computing, vol. 6, pp. 311 312, 2002

N. A. Streitz, J. Geißler, T. Holmer, S. i. Konomi, C. Müller-Tomfelde, W. Reischl, P. Rexroth, P. Seitz, and R. Steinmetz, "i-LAND:An interactive Landscape for Creativity and Innovation," presented at Conference on Human Factors in Computing Systems (CHI’99), Pittsburgh, Pennsylvania, United States, 1999.

K. Koile, K. Tollmar, D. Demirdjian, H. Shrobe, and T. Darrell, "Activity Zones for Context-Aware Computing," in 5th Int. Conf. in Computer Science, A. K. Dey, A. Schmidt, and J. F. McCarthy, Eds. Seattle, WA, USA: Springer-Verlag, 2003, pp. 90-106. Ubiquitous Computing (UbiComp 2003), vol. 2864, Lecture Notes 